\title{
Rashba spin-orbit coupling in the Kane-Mele-Hubbard model
}

\author{
Manuel Laubach, ${ }^{1}$ Johannes Reuther, ${ }^{2}$ Ronny Thomale, ${ }^{1}$ and Stephan Rachel ${ }^{3}$ \\ ${ }^{1}$ Institute for Theoretical Physics, University of Würzburg, 97074 Würzburg, Germany \\ ${ }^{2}$ Department of Physics, California Institute of Technology, Pasadena, California 91125, USA \\ ${ }^{3}$ Institute for Theoretical Physics, Technische Universität Dresden, 01062 Dresden, Germany
}

(Received 18 December 2013; revised manuscript received 10 October 2014; published 27 October 2014)

\begin{abstract}
Spin-orbit (SO) coupling is the crucial parameter to drive topological-insulating phases in electronic band models. In particular, the generic emergence of SO coupling involves the Rashba term which fully breaks the $\mathrm{SU}(2)$ spin symmetry. As soon as interactions are taken into account, however, many theoretical studies have to content themselves with the analysis of a simplified U(1)-conserving SO term without Rashba coupling. We intend to fill this gap by studying the Kane-Mele-Hubbard (KMH) model in the presence of Rashba SO coupling and present the first systematic analysis of the effect of Rashba SO coupling in a correlated two-dimensional topological insulator. We apply the variational cluster approach (VCA) to determine the interacting phase diagram by computing local density of states, magnetization, single particle spectral function, and edge states. Preceded by a detailed VCA analysis of the KMH model in the presence of U(1)-conserving SO coupling, we find that the additional Rashba SO coupling drives new electronic phases such as a metallic regime and a weak topological-semiconductor phase which persist in the presence of interactions.
\end{abstract}

DOI: 10.1103/PhysRevB.90.165136

PACS number(s): 03.65.Vf, 71.27.+a, 73.20.-r

\section{INTRODUCTION}

Since their theoretical prediction [1-4] and experimental discovery [5], topological insulators [6-8] have become one of the most vibrant fields in contemporary condensed matter physics. In two spatial dimensions, the topological insulating state can be interpreted as the spin-type companion of the charge-type integer quantum Hall effect on a lattice. For the quantum spin Hall (QSH) effect, the characteristic feature to drive a given electronic band model into this topologically nontrivial phase is band inversion due to spin-orbit (SO) coupling. Because the kinetic and spin degree of freedom are coupled due to SO coupling, the electronic band structure loses its $\mathrm{SU}(2)$ spin symmetry. Two different types of SO coupling can be distinguished: (i) the intrinsic spin-orbit coupling $V_{\text {ISO }} \sim\left(Z^{4}\right) L^{z} S^{z}$ where the $\mathrm{SU}(2)$ spin group is only broken down to U(1) (i.e., retaining a conserved $S^{z}$ quantum number) and (ii) the Rashba SO coupling $V_{\mathrm{RSO}} \sim \boldsymbol{E} \cdot(\boldsymbol{S} \times \boldsymbol{p})$ which does not retain a conserved continuous subgroup of $\mathrm{SU}(2)$. While the intrinsic SO coupling gives rise to the topological-insulator phase, the Rashba SO coupling itself is unable to induce the nontrivial topology. In any experimental situation, due to the presence of, e.g., a substrate or external electric fields, Rashba SO coupling needs to be taken into account.

As the first microscopic model for topological insulators, the Kane-Mele model was originally proposed to describe the quantum spin Hall effect in graphene [1,2]. Subsequent bandstructure calculations showed, however, that the spin-orbit gap in graphene is so small $[9,10]$ that the QSH effect in graphene is beyond any experimental relevance. Still, Kane and Mele's pioneering proposal for a prototypical topological insulator has triggered an intensive search for possible realizations. In principle, the spin-orbit coupling $\lambda$ can be increased using heavier elements since $V_{\text {ISO }} \propto Z^{4}$ as a function of the atomic coordination number $Z$. Hence, promising proposals include graphene endowed with heavy adatoms such as indium and thallium [11], synthesized silicene [12,13] (monolayers of silicon), molecular graphene [14], honeycomb films of tin [15], monolayers or thin films of the iridium-based honeycomb compounds $X_{2} \mathrm{IrO}_{3}(X=\mathrm{Na}$ or $\mathrm{Li})[16,17]$, and "digital" transition-metal-oxide heterostructures [18]. Alternatively, the Kane-Mele model might be realized by using ultracold atoms in tunable optical lattices [19]. Very recent progress has been made in realizing honeycomb optical lattices [20], as well as non-Abelian gauge fields acting as a synthetic spin-orbit coupling [21-24]. Furthermore, a different route to realize the quantum spin Hall effect on the honeycomb lattice is to induce it by virtue of interactions [25-32].

At the noninteracting level, a Rashba SO term has already been considered in the original work by Kane and Mele where it is shown that the QSH phase of noninteracting fermions is stable with respect to a breaking of $S_{z}$ symmetry. It is also argued that the otherwise-quantized spin Hall conductance will deviate from its quantized value in the presence of a Rashba term $[1,2]$. Later it was explicitly shown that the QSH phase survives the combination of disorder and Rashba spin-orbit coupling but the value of the spin Hall conductance deviates significantly from the quantized value [33].

For the purpose of including interactions in the Kane-Mele model, theoretical approaches have preferably constrained themselves to the exclusive consideration of intrinsic spin-orbit coupling. There are two main reasons for this development. First, some theoretical approaches such as quantum Monte Carlo (QMC) necessitate the U(1) symmetry kept by the intrinsic SO coupling in order to be applicable, i.e., in the case of QMC, to avoid the sign problem. Second, calculating the topological invariant in terms of single-particle Green's functions in the absence of inversion symmetry as implied by Rashba SO coupling is significantly more complicated and often yields an integral form of the Volovik invariant [34], which is not amenable to efficient numerical evaluation. The Kane-Mele model with an onsite Hubbard interaction term and only intrinsic spin-orbit coupling has been usually referred to as the Kane-Mele-Hubbard (KMH) model and has attracted much attention recently; it was investigated from many 
different perspectives [35-55], providing us with a fairly good understanding of its phase diagram: For weak interactions, the topological insulator remains stable and the metallic edge states persist. For intermediate interactions, a phase transition into a magnetically ordered phase occurs. The latter has been shown to exhibit easy-plane antiferromagnetic order [35] and the transition to be of three-dimensional (3D) $X Y$ type $[38,45]$. In the isotropic limit of vanishing spin-orbit coupling, one finds the semimetallic phase (weak interactions) of graphene as well as the Néel antiferromagnet (strong interactions), with the phase transition of regular 3D Heisenberg type [56]. Also, related correlated TI models have been studied [57,58]. (For a review of correlation effects in topological insulators, see Ref. [59].)

Bridging the gap between possible experimental realizations and theoretical modeling, taking into account Rashba SO coupling and interactions in the Kane Mele model is indispensable. Note that the effect of Rashba SO coupling has so far not been investigated in any two-dimensional correlated topological-insulator model (with the exception of the onedimensional edge theory of topological insulators dubbed a helical Luttinger liquid [60-63]). In this article, we employ the variational cluster approach (VCA) $[64,65]$ to investigate the generalized Kane-Mele-Hubbard model in the presence of Rashba spin-orbit coupling. The VCA is an efficient method to investigate interaction effects in correlated electron systems and to obtain effective electronic band structures. Our main results are summarized in Fig. 1. For small Rashba coupling, we find the TI (at small onsite interaction $U$ ) and $X Y$-AFM phases (at large interactions $U$ ) which are also present in the Kane-Mele-Hubbard model without the Rashba coupling. Larger Rashba coupling induces a topologically nontrivial direct-gap-only semiconductor before the system eventually becomes metallic. The $X Y$-AFM phase is found to break down at large Rashba couplings beyond which the evolving magnetic phase cannot be analyzed anymore via VCA due to limited cluster size. Involving the knowledge from alternative approaches, such as pseudofermion functional renormalization group [66,67], this parameter regime is conjectured to be dominated by incommensurate spiral order.

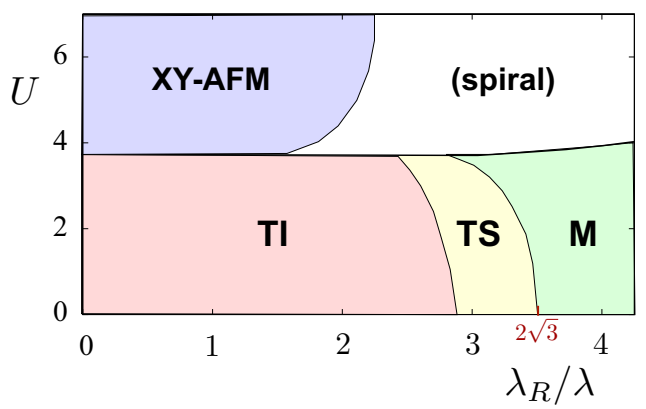

FIG. 1. (Color online) Schematic $U-\left(\lambda_{R} / \lambda\right)$ phase diagram of the full Kane-Mele-Hubbard model for $\lambda=0.2(t=1)$. There are five different phases: topological insulator (TI), weak topological semiconductor (TS), metal (M), easy-plane antiferromagnet ( $X Y$ AFM), and possibly a phase with incommensurate spiral order. For larger $\lambda$ the TS phase becomes broader while for smaller $\lambda$ the TS phase shrinks until it vanishes for $\lambda<0.1$.
The paper is organized as follows: In Sec. II, we introduce the Kane-Mele-Hubbard model and briefly describe the variational cluster approach (VCA). In Sec. III, we establish a first VCA benchmark by showing results for the KMH model in the absence of Rashba spin-orbit coupling. This scenario serves as a prototypical framework to illustrate various subtle issues in the VCA approach such as cluster dependence, where details are delegated to Appendix A. Subsequently, the results for the $\mathrm{KMH}$ model in the presence of finite Rashba SO coupling are presented in Sec. IV. In Sec. V, we conclude that the nontrivial phases of the Kane-Mele model emerging due to Rashba SO coupling persist in the presence of interactions, and that the interplay of interactions and Rashba SO coupling establishes a promising direction of study in theory and experiment.

\section{MODEL AND METHODOLOGY}

\section{A. Kane-Mele Hubbard model with Rashba spin-orbit coupling}

The Kane-Mele-Hubbard model is governed by the Hamiltonian

$$
\begin{aligned}
\mathcal{H}= & -t \sum_{\langle i j\rangle \sigma} c_{i \sigma}^{\dagger} c_{j \sigma}+i \lambda \sum_{\langle\langle i j\rangle\rangle \alpha \beta} c_{i \alpha}^{\dagger} v_{i j} \sigma_{\alpha \beta}^{z} c_{j \beta} \\
& +i \lambda_{R} \sum_{\langle i j\rangle \alpha \beta} c_{i \alpha}^{\dagger}\left(\sigma_{\alpha \beta} \times \boldsymbol{d}\right)_{z} c_{j \beta}+U \sum_{i} n_{i \uparrow} n_{i \downarrow} .
\end{aligned}
$$

The operator $c_{i \alpha}$ annihilates a particle with spin $\alpha$ on site $i, t$ is the hopping amplitude (which we set to unity, $t \equiv 1$, throughout the paper), $\lambda$ is the intrinsic spin-orbit coupling, $\lambda_{R}$ is the amplitude of the Rashba SO coupling, $U$ parametrizes the local Coulomb (Hubbard) interactions, and $v_{i j}= \pm 1$ depending on whether the electron traversing from $i$ to $j$ makes a right $(+1)$ or a left $(-1)$ turn [Fig. 2(a)]. As usual, $\langle i j\rangle$ indicates that $i$ and $j$ are nearest-neighbor sites while $\langle\langle i j\rangle\rangle$ refers to second-nearest neighbors. The vector $\boldsymbol{d}$ points from site $i$ to site $j$ and corresponds to the nearest-neighbor vectors $\boldsymbol{\delta}_{i},(i=1,2,3)$ [Fig. 2(b)]; $\sigma^{\mu}(\mu=x, y, z)$ denotes the three Pauli matrices corresponding to the spin degree of freedom. The explicit spin dependence of the Rashba SO term, $(\boldsymbol{\sigma} \times \boldsymbol{d})_{z}$, is visualized in Fig. 2(b). The spin-orbit term $\propto \lambda$ breaks the SU(2) symmetry down to U(1), the Rashba term $\propto \lambda_{R}$ breaks the remaining $\mathrm{U}(1)$ spin symmetry down to $\mathbb{Z}_{2}$. It also explicitly breaks the spatial inversion symmetry. The Rashba spin-orbit term as a part of the original Kane-Mele model has so far generally been neglected in studies of the interacting scenario. Note that, in the original work by (a)

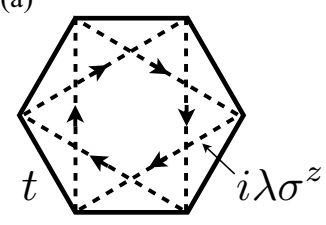

(b)

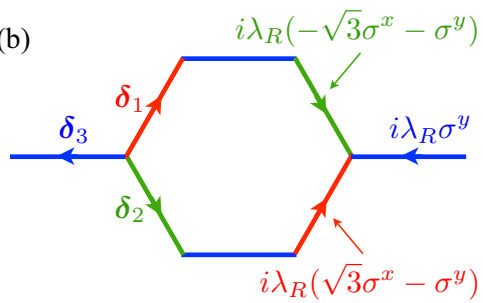

FIG. 2. (Color online) (a) Illustration of the hopping term $\propto t$ and the intrinsic SO term $\propto i \lambda \sigma^{z}$. (b) Illustration of the nearest-neighbor vectors $\boldsymbol{\delta}_{i}(i=1,2,3)$ and of the Rashba SO term $\propto i \lambda_{R}$ with different spin dependencies in different hopping directions $\boldsymbol{\delta}_{i}$. 
Kane and Mele, a staggered sublattice potential (Semenoff mass) has also been discussed which we will not elaborate on further in the following. This term is particularly useful to probe the transition from a topological band insulator phase into a trivial band insulator phase [1,2,68-71] but does not yield distinctly new phases, which is the focus of our investigations in the following.

\section{B. Variational cluster approach}

\section{Method}

The zero-temperature variational cluster approach (VCA) [72] is based on the self-energy functional theory $[65,73]$, which provides an efficient numerical technique for studying strongly correlated systems, especially in the presence of different competing, potentially long-ranged orders. VCA simplifies the lattice problem, as defined in Eq. (1), to an exactly solvable problem defined in a reference system consisting of decoupled finite-size clusters. The thermodynamic limit is recovered by reintroducing the intercluster hopping to the decoupled cluster via a nonperturbative variational scheme based on self-energy functional theory. The VCA has been successfully applied to many interesting problems, including the high- $T_{c}$ cuprates $[74,75]$ and correlated topological insulators [41]. In particular, this method is suitable for our current study since the topologically nontrivial properties of the $\mathbb{Z}_{2}$ topological insulators are accounted for appropriately. By construction, the VCA becomes exact in the limit of $U \rightarrow 0$. Hubbard onsite interactions might give rise to competing phases (such as magnetic order) which can be accurately described by the VCA grand potential.

In the self-energy functional theory, the grand potential of a system defined by a Hamiltonian $H=H_{0}(\mathbf{t})+H_{1}(\mathbf{U})$ is written as a functional of the self-energy $\Sigma$ :

$$
\Omega[\Sigma]=F[\Sigma]+\operatorname{Tr} \ln \left(G_{0}^{-1}-\Sigma\right)^{-1},
$$

where $F[\Sigma]$ is the Legendre transform of the LuttingerWard functional and $G_{0}=(\omega+\mu-\mathbf{t})^{-1}$ is the noninteracting Green's function. It can be shown that the functional $\Omega[\Sigma]$ becomes stationary at the physical self-energy, i.e., $\delta \Omega\left[\Sigma_{\text {phys }}\right]=$ 0 [72]. Because the Luttinger-Ward functional is universal, it has the same interaction dependence for systems with any set of $\mathbf{t}^{\prime}$ as long as the interaction $\mathbf{U}$ remains unchanged. Note that the functional $\Omega[\Sigma]$ itself is not approximated by any means; we restrict, however, the "parameter" space of possible self-energies to the self-energies of the reference system. Thus, the stationary points are obtained from the self-energy $\Sigma^{\prime}=\Sigma\left[\mathbf{t}^{\prime}\right]$ of a system defined by the Hamiltonian $H^{\prime}=H_{0}\left(\mathbf{t}^{\prime}\right)+H_{1}(\mathbf{U})$, which we label as reference system. Let us define $V=\mathbf{t}-\mathbf{t}^{\prime}$. Now we are able to conveniently define the VCA-Green's function,

$$
G_{\mathrm{VCA}}^{-1}=G^{\prime-1}-V
$$

In terms of the reference system, the VCA grand potential is calculated more conveniently as

$$
\Omega\left[\Sigma^{\prime}\right]=\Omega^{\prime}+\operatorname{Tr} \ln \left(G_{0}^{-1}-\Sigma^{\prime}\right)^{-1}-\operatorname{Tr} \ln \left(G^{\prime}\right),
$$

with $\Omega^{\prime}, \Sigma^{\prime}$, and $G^{\prime}$ denoting the grand potential, the selfenergy and the Green's function of the reference system, respectively. The reference system is chosen such that it can be treated exactly. Here, we choose an array of decoupled clusters with open boundary conditions and calculate $\Omega^{\prime}, \Sigma^{\prime}$, and $G^{\prime}$ via exact diagonalization. While the correlation beyond the reference system size are included on a mean-field level, the short-range correlations within the reference system are fully taken into account in the VCA, resembling related (cluster) dynamical mean-field theory approaches.

\section{Cluster size and shape}

Since a spinful Hubbard model involves four basis states for each lattice site, we are generally restricted to rather small clusters with a maximum of ten sites [Fig. 3(b)]. Furthermore, the choice of the reference system, i.e., the cluster shape and size, is constrained by the requirement that the honeycomb lattice needs to be fully covered, either by using periodic boundary conditions (PBCs) - as realized on a torus-or cylindrical boundary conditions. We consider six-, eight-, and ten-site clusters in the case of PBCs and eight-site clusters for cylindrical boundary conditions with zigzag edges (Fig. 3). [Note that the six- and ten-site clusters could also be used for ribbons (cylinders) with armchair edges which is not further considered here; see also Ref. [38].] While one generally expects to obtain more accurate results with a larger cluster, the effect of the lattice partitioning, i.e., the cluster dependence, is rather strong. We therefore extract our physical results from the joint consideration of all cluster sizes reachable by VCA, which is indispensable to obtain physically meaningful results from finite-cluster approaches in general.

In the topological-insulator phase we explore the edge states connecting the valence and conduction bands of the system. These edge states typically penetrate a few unit cells into the bulk. If the ribbon height (i.e., the distance between upper and lower edges) does not exceed a few unit cells it might happen that the penetrating edge states from the upper and lower edges couple to each other and gap out. To avoid this, we have to make sure that the ribbon height is sufficiently large; we build a supercluster which consists of $n$ normal clusters (as described (a)

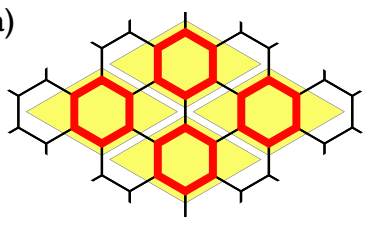

(c)

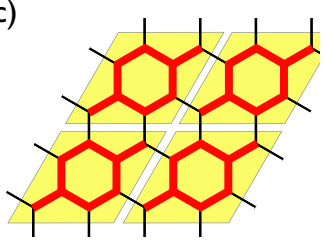

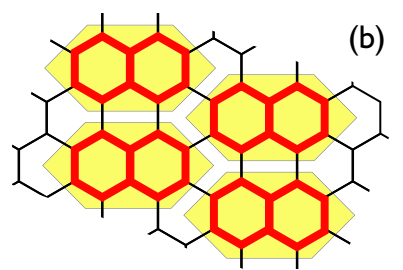

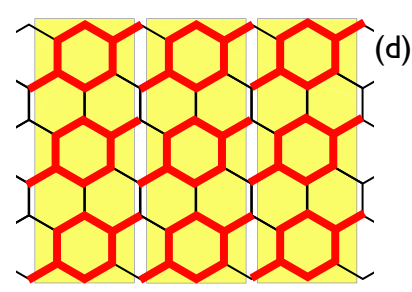

(b)
FIG. 3. (Color online) Honeycomb lattice covered with single clusters in VCA: (a) six-site clusters (PBC). (b) Ten-site clusters (PBC). (c) Eight-site clusters (PBC). (d) Honeycomb ribbon (cylinder) covered with eight-site clusters. 
above) and stack them on top of each other as illustrated in Fig. 3(d). The supercluster corresponds to the unit cell of the effectively one-dimensional superlattice and is defined by the tridiagonal matrix

$$
G^{\prime-1}=\left(\begin{array}{cccccc}
G_{1}^{\prime-1} & t_{1,2} & & & & \\
t_{2,1} & G_{2}^{\prime-1} & t_{2,3} & & & \\
& t_{3,2} & G_{3}^{\prime-1} & t_{3,4} & & \\
& & \ddots & \ddots & \ddots & \\
& & & t_{n-1, n-2} & G_{n-1}^{\prime-1} & t_{n-1, n} \\
& & & & t_{n, n-1} & G_{n}^{\prime-1}
\end{array}\right),
$$

where $G^{\prime}$ is the Green's function of the supercluster with the dimension $2 L_{c} \times n, G_{i}^{\prime}$ are the cluster Green's functions, and $t_{i, i+1}$ is the hopping matrix connecting the two cluster Green's functions $G_{i}^{\prime}$ and $G_{i+1}^{\prime} ; L_{c}$ is the number of cluster sites. To separate edge states from the the upper and lower edges we stack at least eight clusters to form a supercluster from which we compute the single-particle spectral function (displaying the edge states). The single-particle spectral function $A(k, \omega)$ is defined as in the standard case of PBCs via

$$
A(k, \omega)=-\frac{1}{\pi} \operatorname{Im}\left\{G_{\mathrm{VCA}}(k, \omega)\right\},
$$

where the VCA-Green's function depends on the momentum $k$ retained by the circumferential direction of the cylinder.

\section{Symmetry-breaking Weiss fields}

In quantum cluster approaches (and dynamical mean-field theory) manifestations of spontaneous symmetry breaking for finite-size clusters is resolved by introducing artificial meanfield-like Weiss fields of the form

$$
H_{X-\mathrm{AF}}=h^{x} \sum_{i \alpha \beta}\left(a_{i \alpha}^{\dagger} \sigma_{\alpha \beta}^{x} a_{i \beta}-b_{i \alpha}^{\dagger} \sigma_{\alpha \beta}^{x} b_{i \beta}\right),
$$

where the operator $a_{i}\left(b_{i}\right)$ acts on sublattice $A(B)$. Equation (7) is the simplest example of an antiferromagnetic Weiss field with Néel order in the $x$ direction (in plane). Given an external Weiss field for a certain order parameter, a stable magnetic solution is characterized by a stationary point in the grand potential at a finite field strength. Furthermore, in order to represent the physical ground state, such a stationary point needs to have a lower energy than the zero-field solution. In principle, similar to a mean-field treatment, this procedure needs to be repeated for all possible configurations of Weiss fields. The order parameter can then be determined from the magnetic solution with the lowest energy. The cluster decomposition of the lattice, however, restricts the possible choices of Weiss fields to those which are compatible with the cluster size and shape, i.e., a Weiss field needs to have the same periodicity as the array of clusters. Typically, for a given cluster only a few types of magnetic order may be investigated. For example, a Néel pattern cannot be implemented on a three-site cluster. Likewise, incommensurate spiral order is incompatible with any finite cluster.

\section{Variation of single-particle parameters}

The variational procedure of VCA works such that the amplitudes of every single-particle term as well as the chemical potential $\delta \mu$ need to be varied. It is well established, however, that for practical purposes the variation of $\delta \mu$ is often sufficient and the additional variation of, say, the hopping $\delta t$ does not lead to a new stationary point. For the KMH model, in principle we have to vary not only the chemical potential, but also the hopping, spin-orbit coupling, and Rashba terms independently. In Appendixes A and B, we show exemplarily the difference between (i) variation of $\delta \mu$, (ii) variation of $\delta \mu$ and $\delta t$, (iii) variation of $\delta \mu, \delta t$, and $\delta \lambda$, as well as (iv) variation of additional antiferromagnetic Weiss fields. Essentially, we find that variation of $\delta t$ has a significant effect on the phase diagrams, including magnetic phase transitions. Additional variation of $\delta \lambda$ or $\delta \lambda_{R}$, respectively, does not seem to influence the variational procedure. Still, performing VCA on the honeycomb lattice with variation of $\delta \mu$ only might lead to numerical artifacts and should be avoided. Further details are illustrated in Appendixes A and B.

\section{KANE-MELE-HUBBARD MODEL WITHOUT RASHBA SO COUPLING $\left(\lambda_{R}=0\right)$}

\section{A. Topological insulator

$$
\text { 1. } \mathbb{Z}_{2} \text { invariant }
$$

In the presence of inversion symmetry the topological invariant can be conveniently calculated by probing bulk properties only, which is even applicable in the interacting case. In particular, within VCA this can be achieved for any cluster size.

Expressing topological invariants in terms of single-particle Green's functions was pioneered by Volovik [34]; more recently, Gurarie [76] conveniently reformulated Volovik's invariant for the field of topological insulators. Recently, Wang et al. [77,78] derived simplified expression for inversionsymmetric Hamiltonians. The $\mathbb{Z}_{2}$ topological invariant relevant for topological insulators is computed from the full interacting Green's function through a Wess-Zumino-Witten term [77], motivated from the concept of dimensional reduction in topological field theory $[7,79]$.

In the presence of inversion symmetry (i.e., when $\lambda_{R} \equiv 0$ and antiferromagnetic order is absent), we follow Wang et al.to compute the topological invariant formula [78] via the parity eigenvalues of the Green's function obtained within VCA at the time-reversal invariant momenta (TRIM) $\boldsymbol{\Gamma}_{i}$ and zero energy. The Green's function is a $N \times N$ matrix with $N=2 L_{c}$, where $L_{c}$ is the number of sites per cluster. Both $G$ and $G^{-1}$ can be diagonalized, yielding

$$
G(i \omega, \boldsymbol{k})^{-1}|\alpha(i \omega, \boldsymbol{k})\rangle=\mu_{\alpha}(i \omega, \boldsymbol{k})|\alpha(i \omega, \boldsymbol{k})\rangle,
$$

with $\mu_{\alpha} \in \mathbb{C}$. The Green's function matrix $G(i \omega, \boldsymbol{k})$ has the same eigenvectors $|\alpha(i \omega, \boldsymbol{k})\rangle$ but the inverse eigenvalues $\mu_{\alpha}^{-1}(i \omega, \boldsymbol{k})$. The states at the TRIMs, $\left|\alpha\left(i \omega, \boldsymbol{\Gamma}_{i}\right)\right\rangle$, are simultaneous eigenstates of $G$ and $P$ and satisfy [78]

$$
P\left|\alpha\left(i \omega, \boldsymbol{\Gamma}_{i}\right)\right\rangle=\eta_{\alpha}\left|\alpha\left(i \omega, \boldsymbol{\Gamma}_{i}\right)\right\rangle .
$$

Since $\mu_{\alpha}\left(0, \Gamma_{i}\right)$ is real, one can distinguish between positive $\left[\mu_{\alpha}\left(0, \boldsymbol{\Gamma}_{i}\right)>0\right]$ and negative $\left[\mu_{\alpha}\left(0, \boldsymbol{\Gamma}_{i}\right)<0\right]$ eigenvalues, 

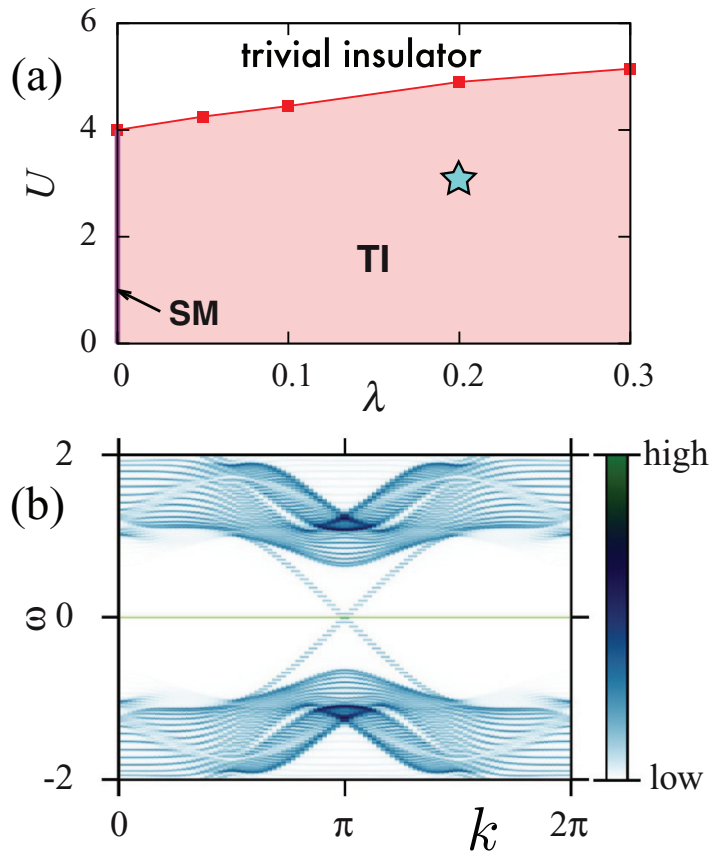

FIG. 4. (Color online) (a) Phase boundary in $U-\lambda$ plane between topological insulator and trivial band insulator ("nonmagnetic" solution) obtained by a periodic eight-site cluster computation of the $\mathbb{Z}_{2}$ invariant. (b) Edge spectrum in the TI phase obtained for cylindrical geometry; parameters $\left(\lambda=0.2, U=3, \lambda_{R}=0\right)$ correspond to the light-blue star in the phase diagram in panel (a). Panels (a) and (b) show complementary approaches to detect the topological-insulating phase.

denoted as R-zeros and L-zeros, respectively. This allows us to define the topological invariant $\Delta$ via

$$
(-1)^{\Delta}=\prod_{\text {R-zero }} \eta_{\alpha}^{1 / 2}= \pm 1 .
$$

In Fig. 4(a) we show the $U-\lambda$ plot of this invariant. Note again that $\Delta$ cannot be calculated when an antiferromagnetic Weiss field is present due to breaking of inversion symmetry. As a consequence, in VCA we independently investigate the magnetically ordered regime. The onset of a finite magnetization likewise sets the boundary for which the topological character of the insulating state vanishes.

\section{Edge states}

As an alternative to a bulk measurement of the topological invariant, the topological-insulator phase can also be identified by detecting the helical edge states which are a hallmark of $\mathbb{Z}_{2}$ topological insulators considered here. This is accomplished by solving the Hamiltonian (1) on a cylindric geometry as explained in the previous section. This method is reliable and is also applicable when the computation of the topological bulk invariant is too complicated, such as for finite Rashba SO coupling addressed later. In Fig. 4(b) the single-particle spectral function $A(k, \omega)$ defined for a ribbon geometry is shown $\left(\lambda=0.2, \lambda_{R}=0, U=4\right)$. In the effectively-onedimensional Brillouin zone, one clearly sees a band gap between the upper and lower bands, which are connected by helical edge states crossing at the TRIM $k=\pi$.
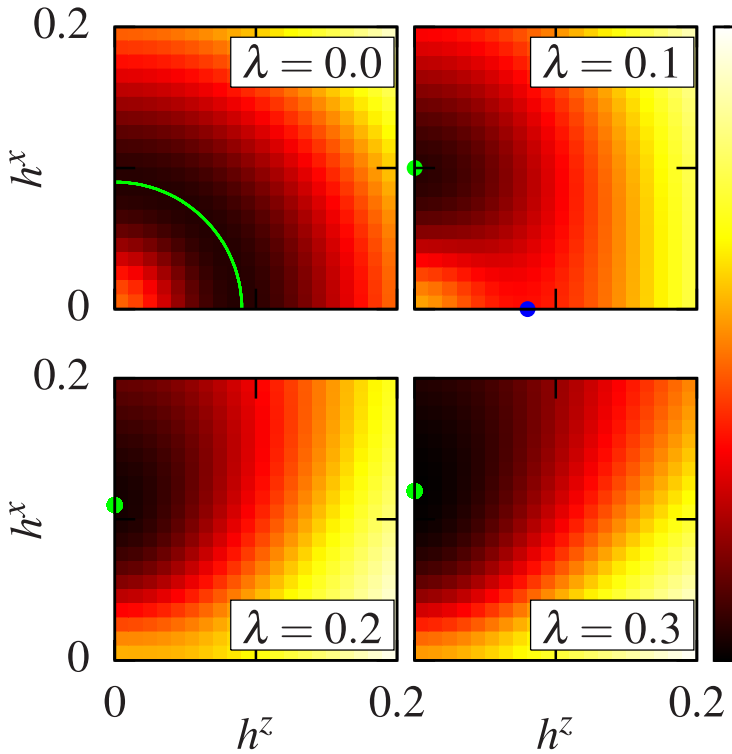

FIG. 5. (Color online) Heat map of the grand potential $\Omega\left(h^{x}, h^{z}\right)$ as a function of antiferromagnetic Weiss fields $h^{x}$ and $h^{z}$ for various values of $\lambda$. All plots haven been obtained for the six-site cluster and $U=6$. Global minima of $\Omega$ are indicated by green points (lines). For $\lambda=0.1$ we find a second stationary point (blue point) which is a saddle point at finite $h^{z} \neq 0$ with higher energy.

\section{B. $X Y$ antiferromagnet}

For $\lambda \rightarrow 0$ the Hamiltonian (1) becomes invariant under $\mathrm{SU}(2)$ spin rotations and the antiferromagnetic Néel order is isotropic. Finite SO coupling $\lambda \neq 0$ drives the system into an easy-plane antiferromagnet with an ordering vector in the $x y$ lattice plane [35], which has been confirmed by QMC [36,39], VCA [41], and pseudofermion functional renormalization group [66]. In order to compute the magnetic phase diagram within VCA, we apply antiferromagnetic Weiss-fields in $x$ and $z$ direction for various values of $\lambda$.

For $\lambda=0$ we find a circle of degenerate minima in the $h^{x}-h^{z}$ plane, indicating isotropic magnetic order. For finite $\lambda>0$, this degeneracy is lifted and magnetic order in the $x$ direction is energetically preferred. For small $\lambda=0.1$ there is an additional stable solution (a saddle point in $\Omega$ indicated by the blue point in Fig. 5 right-top panel) corresponding to a magnetization in the $z$ direction. This solution, however, is not a global minimum in $\Omega$ and the system is still an easyplane antiferromagnet. For larger $\lambda$, this metastable solution disappears. In total, the VCA confirms the established results about magnetic order in the KMH.

\section{Phase diagram}

As the final result, the interacting $U-\lambda$ phase diagram exhibits a semimetal for $\lambda=0$ which is detected via a linear density of states near the Fermi level. It transcends into a topological-insulator phase for finite $\lambda$ up to moderate interaction strengths. For stronger interactions, the system acquires $X Y$ antiferromagnetic order. Obtaining a phase diagram such as Fig. 6 via a quantum cluster approach is challenging: (i) Stabilizing semimetals within real-space quantum cluster methods is rather involved; in particular 


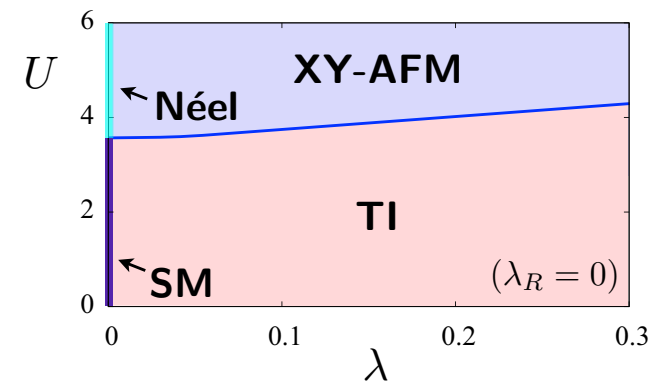

FIG. 6. (Color online) Schematic phase diagram of the KaneMele-Hubbard model $\left(\lambda_{R}=0\right)$ as obtained from VCA.

the six-site cluster may suffer from artifacts of the lattice partitioning. (ii) Clusters which do not have the shape of closed honeycomb rings underestimate the critical interaction strength $U_{c}$ associated with the onset of magnetization. (iii) Exclusive variation of the chemical potential might lead to an erroneous nonmagnetic insulator phase up to small intrinsic spin-orbit coupling [41]. In our analysis, where we also varied the hopping in order to minimize the grand potential, we could not find this nonmagnetic insulator phase. Note that this erroneous nonmagnetic insulator phase was linked to a proposed quantum spin-liquid phase. Recently, it was shown by using large-scale QMC calculations that there is no such spin liquid on the honeycomb lattice $[56,80]$, being in perfect agreement with our analysis. [For an extensive discussion and details about (i)-(iii) we refer the interested reader to Appendix A.] The analysis done so far shows that a careful multisize cluster analysis has to be employed in order to determine an artifact-free physical phase diagram. This prepares us for our subsequent investigations of the $\mathrm{KMH}$ model in the presence of Rashba SO coupling studied in the next section.

\section{KANE-MELE-HUBBARD MODEL INCLUDING RASHBA SO COUPLING $\left(\lambda_{R}>0\right)$}

In their seminal papers, Kane and Mele showed that the topological-insulator phase persists until $\lambda_{R}=2 \sqrt{3} \lambda$ where the gap closes and the system enters a metallic phase [1,2]. They computed the $\mathbb{Z}_{2}$ invariant to explore the corresponding phase diagram. In their work, they considered rather small values of SO coupling such as $\lambda=0.03$ or 0.06 , and in general $\lambda \ll t$. For a description of graphene, which was the original intention of this work, such small SO coupling seemed to be realistic. However, with regard to the many different candidate systems potentially realizing the quantum spin Hall effect in a honeycomb lattice compound which have been proposed in the meantime, it is justified to consider larger spin-orbit coupling such as $\lambda=0.2$. It turns out that, for sufficiently large $\lambda \geqslant 0.1$ and $\lambda_{R}$ close to the predicted phase transition at $\lambda_{R}=2 \sqrt{3} \lambda$, the system is not gapped anymore. The Rashba SO coupling bends the bands such that there is no full gap. On the other hand, there is always a direct gap for each wave vector $k$, i.e., the conductance and valence bands neither touch nor cross each other-this is the reason why the topological invariant (computed for $U=0$ ) labels this region as a topological insulator. In fact, in this "metallic" region the edge states are well defined and clearly visible [see the second-right panel in Figs. 7 and 8(b)]. At each momentum $k$ the system has a gap, but globally the system is gapless. Therefore we call this region a weak topological-semiconductor phase where "semiconductor" refers to a direct-gap-only insulating phase. In the presence of disorder individual $k$ values cannot be distinguished anymore, leading to the attribute weak, as the phase breaks down in the presence of disorder. Still, this phase is stable for the clean case in the presence of interactions, as we explicate below.

\section{A. Weak-to-intermediate interactions}

For $\lambda<0.1$, we only find TI and metallic phases at $U=0$, which persist for moderate interaction strength. Fixing $\lambda=0.2$ we find three different phases at $U=0$ : TI, weak topologicalsemiconductor (TS) phase, and metal [see Figs. 8(a) and 8(b)]. The TS phase is stable with respect to interactions; see Fig. 8(c). To gain further insight, we compute single-particle spectral functions on cylindrical geometry (using the eight-site cluster) to determine the edge-state spectrum (see Fig. 9). For $\lambda=0.2$ and $\lambda_{R}=0.6$, the TS phase is stable up to moderate values of $U$. At around $U=4$ the system enters a magnetically ordered phase. Upon further increasing $U$ the bulk gap increases rapidly; however, no edge states connect the valence and conductance bands anymore, indicating the trivial topology of the magnetic phase.

We perform an additional test to verify that the two modes crossing at $k=\pi$ in Fig. $9(U=0$ and $U=2)$ are indeed edge states: we repeat the computation of the single-particle spectral function $A(k, \omega)$ on a cylindrical geometry but with additional links connecting the two edges of the cylinder. These additional links are chosen such that they are compatible with the band structure of the KMH model. As such, moving from
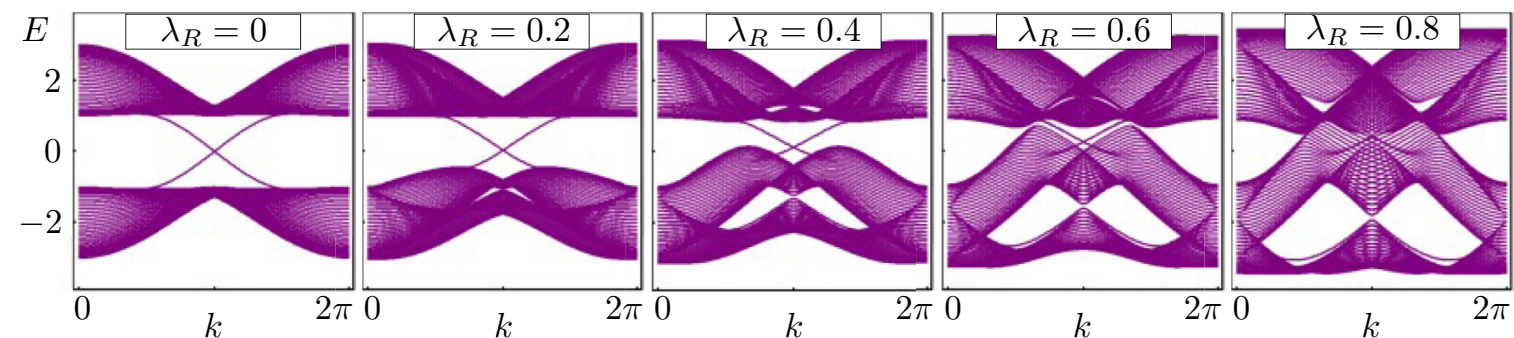

FIG. 7. (Color online) Single-particle spectra on a cylinder geometry for $U=0, \lambda=0.2$, and different values of $\lambda_{R}$. From left to right: $\lambda_{R}=0,0.2,0.4,0.6$, and 0.8 . The spectra interpolate from a topological-insulating phase $\left(\lambda_{R}=0,0.2\right.$, and 0.4$)$ to a metallic phase $\left(\lambda_{R}=0.8\right)$ In between, for $\lambda_{R}=0.6$ we find an additional weak topological-semiconductor phase (see also Fig. 8). 

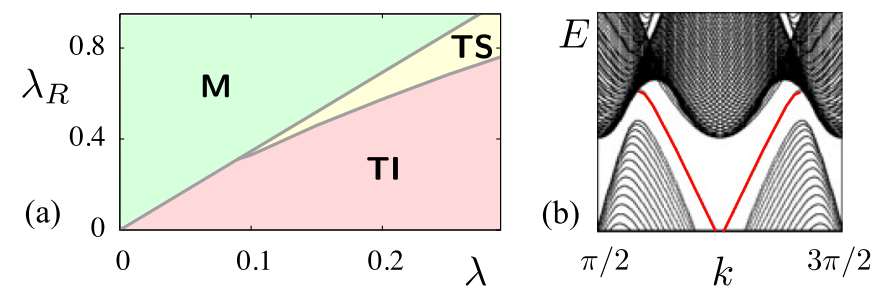

(c)

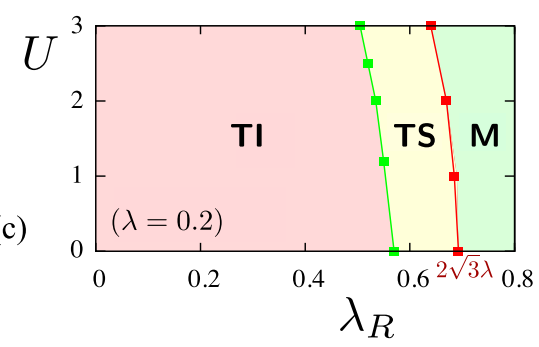

FIG. 8. (Color online) (a) $\lambda_{R}-\lambda$ phase diagram for the noninteracting Kane-Mele model displaying the TI, metal (M), and topological-semiconductor (TS) phase. (b) Zoom into the edge spectrum for $\lambda=0.2, \lambda_{R}=0.6, U=0$ shown in Fig. 7. (c) $U-\lambda_{R}$ phase diagram for $\lambda=0.2$ in the nonmagnetic regime: the weak TS phase persists in the presence of interactions.
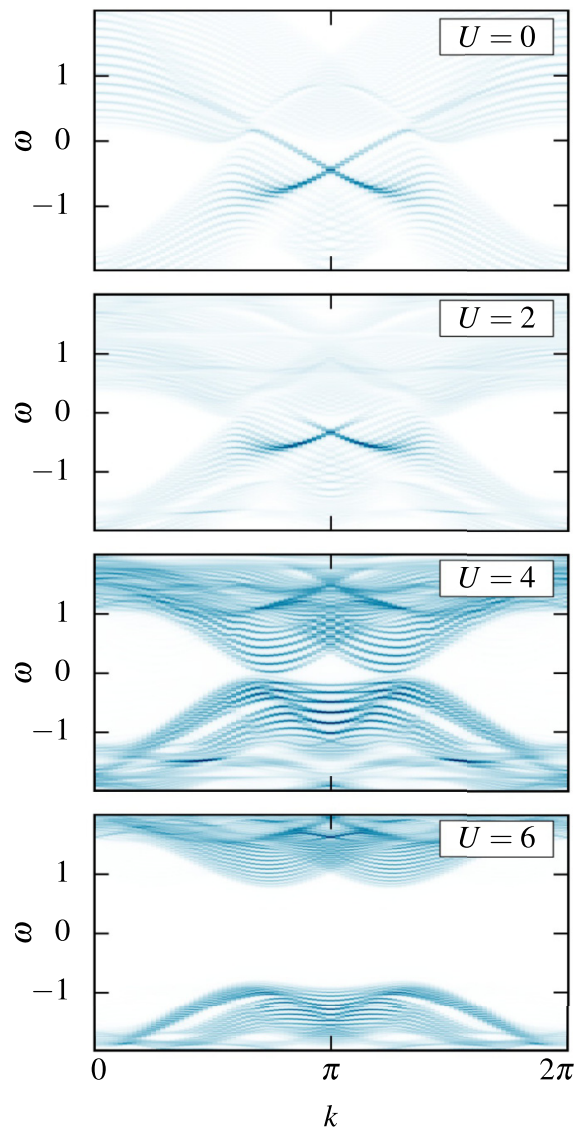

FIG. 9. (Color online) Spectral function $A(k, \omega)$ on cylindrical geometry [as defined in Eq. (6)] for $\lambda=0.2, \lambda_{R}=0.6$, and various values of $U$. For better illustration, only the weights of the outermost sites on the cylinder are taken into account. From top to bottom: $U=0,2,4$, and 6 . For $U=0$ and $U=2$ we find the weak TS phase; for $U=4$ and $U=6$ we find a magnetically ordered insulating phase. a cylindric to a toroidal geometry, the bulk spectra should be unchanged with the only difference being that the edges have disappeared, which is exactly what we find.

\section{B. Strong interactions and magnetic order}

For finite $\lambda>0$ and $\lambda_{R}=0$, the magnetic region of the phase diagram is an $X Y$ antiferromagnet as discussed above. Treating the Rashba term as a small perturbation leaves the magnetic phase unchanged. Thus we expect an $X Y$-AFM in the weak- $\lambda_{R}$ region.

First, we use the six-site cluster and compute the grand potential $\Omega$ as a function of $h^{x}$ and $h^{y}$. As expected we find the $X Y$-AFM. $\Omega$ as a function of $h^{x}$ and $h^{y}$ shows a perfect circle at finite Weiss fields $h^{x / y}$ (Fig. 10).

For the six-site cluster, the saddle point associated with the $X Y$-AFM phase is found at decreasing Weiss fields $h^{x / y}$ when we increase the Rashba coupling. For $\lambda_{R}=0.3$ (at fixed $\lambda=0.1$ ), we do not find any magnetic solution anymore (see lower panels in Fig. 10). This implies that there is either a true nonmagnetic insulator phase or there is a magnetically ordered phase which cannot be detected within VCA. For instance, this is the case for incommensurate spiral order, where the Weiss field is incompatible with the cluster partitioning. A spiral phase is likely to occur since the spin Hamiltonian [i.e., the Hamiltonian obtained in the strong-coupling limit $U \rightarrow \infty$ of Eq. (1)] contains terms of Dzyaloshinskii-Moriya type [66]. Recently, spiral order was also found in a Kane-Mele-type model [16], with multidirectional SO coupling in the presence of strong interactions $[66,81,82]$.

In principle, we cannot rule out the existence of the nonmagnetic insulator phase for large $U$ and large Rashba spin-orbit coupling. The existence of such a phase would be exciting, in particular, since it could be related to a recently proposed fractionalized quantum spin-Hall phase (dubbed $\left.\mathrm{QSH}^{\star}\right)$ [83].

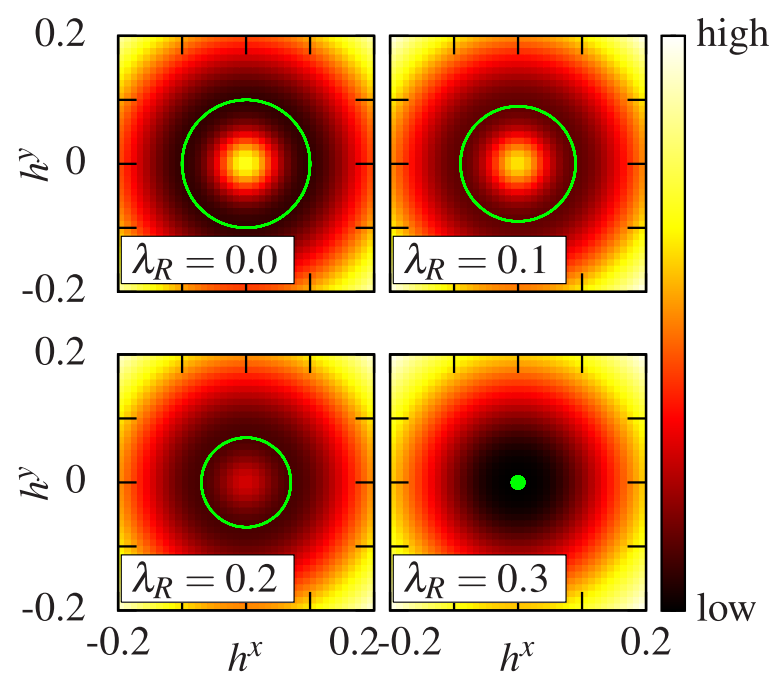

FIG. 10. (Color online) Heat map of the grand potential as a function of antiferromagnetic Weiss fields $\Omega\left(h^{x}, h^{y}\right)$. On the six-site ring-shaped cluster we find easy-plane AFM order for $\lambda_{R}<0.3$ (at $\lambda=0.1$ and $U=6$ ). For larger Rashba coupling we do not find any saddle points at finite Weiss fields. 


\section{Phase diagram}

As the final result of this section and this paper, the $U-\lambda_{R}$ phase diagram contains, for moderate Rashba SO coupling $\lambda_{R}$, a TI phase (weak interactions) and an $X Y$-AFM phase (strong interactions). Stronger Rashba SO coupling drives the TI into a metallic phase. If the intrinsic SO coupling $\lambda$ is sufficiently large $(\lambda \geqslant 0.1)$ an additional weak topological-semiconductor phase emerges between the TI and the metallic phase. In the strong-interaction regime, we do not find a magnetic solution whose unit cell would be consistent with the available cluster sizes in VCA, a regime which is hence likely to host incommensurate spiral magnetic order. All these findings cumulate in the schematic phase diagram shown in Fig. 1.

\section{CONCLUSIONS}

We investigated the effect of Rashba spin-orbit coupling in the Kane-Mele-Hubbard model as a prototypical correlated topological insulator. We applied the variational cluster approach and determined the phase diagram via the computation of local density of states, magnetization, single-particle spectral function, and edge states to detect the topological character. The topological-insulating phase persists in the presence of Rashba spin-orbit coupling and interactions. Furthermore, in the strong-coupling regime, the Rashba term induces magnetic frustration which leads to incommensurability effects in the magnetic fluctuation profile and is conjectured to predominantly give rise to spiral magnetic phases. Rashba spin-orbit coupling also gives rise to peculiar metallic phases. We find a weak topological-semiconductor phase, for a wide range of Hubbard interaction strengths as well as intrinsic and Rashba spin-orbit couplings. It will be exciting to investigate some of these effects in future experiments which exhibit the Rashba term due to external fields or intrinsic environmental effects.

\section{ACKNOWLEDGMENTS}

The authors acknowledge discussions with Karyn Le Hur, Martin Hohenadler, Fakher F. Assaad, Andreas Rüegg, Motohiko Ezawa, Tobias Meng, Michael Sing, Jörg Schäfer, and Matthias Vojta. We thank the LRZ Munich and ZIH Dresden for generous allocation of CPU time. M.L. is supported by the DFG through FOR 1162. J.R. acknowledges support by the Deutsche Akademie der Naturforscher Leopoldina through grant LPDS 2011-14. R.T. is supported by the ERC starting grant TOPOLECTRICS of the European Research Council (ERC-StG-2013-336012). S.R. is supported by the DFG through FOR 960, the DFG priority program SPP 1666 "Topological Insulators," and by the Helmholtz Association through VI-521. We thank the Center for Information Services and High Performance Computing (ZIH) at TU Dresden for generous allocations of computer time.

\section{APPENDIX A: CLUSTER ANALYSIS OF KMH MODEL $\left(\lambda_{R}=0\right)$}

\section{Semimetallic phase for $\lambda=0$}

The semimetal phase of the honeycomb lattice is more sensitive to the lattice partitioning as compared to other phases and lattices. As we discuss in the following, cluster size and shape influence the results. A six-site cluster [having the shape of a single hexagon; see Fig. 3(a)] immediately opens a single-particle gap for $U>0$. In contrast, an eight-site cluster [a hexagon with two additional legs; see Fig. 3(c)] provides an extended semimetallic region before the gap opens at $U_{c}$. It is insightful to further analyze the features of VCA for the different cluster sizes. Let us consider the six-site cluster in the following. As mentioned in Sec. II A, one solves the small cluster exactly by using exact diagonalization (ED). In the absence of any SO coupling, we expect a semimetallic region for $0<U \leqslant U_{c}$ where the effect of the interactions just causes renormalization of the Fermi velocity of the system. In case of our small cluster, we expect a renormalization of the hopping parameter $t$ which we call $\tilde{t}$. In the next step of the VCA, an (infinitely) large lattice is covered by these ED clusters, and the clusters are coupled by the hoppings of the original noninteracting band structure, i.e., by $t$. Hereby, the intracluster hoppings may be varied in order to find a stationary point in the grand potential. That is, for finite but not-toolarge values of $U$, we effectively obtain a plaquette-isotropic honeycomb model [38], as shown in Fig. 11(a). Remarkably, for nearest-neighbor hoppings the band gap opens immediately when $\tilde{t} \neq t$. Indeed, an infinitesimal anisotropy opens an infinitesimal gap [38]. In agreement with this idea, we find that the VCA method using the six-site cluster finds a semimetal only for $U=0$. For any finite $U$ a nonmagnetic insulator phase appears [Fig. 11(d)].

We also tested the influence of bath sites for the six-site cluster [84]. For each correlated site we added one bath site (resulting in an effective twelve-site cluster computation). We still found instant opening of the single-particle gap, although the size of the gap was reduced compared to the results without bath sites (in agreement with Ref. [84]). Variation of the intracluster hoppings $t$ seems to have a similar effect as adding bath sites. Variation of the hoppings and adding bath sites simultaneously further decreases the size of the single-particle gap; it does not change, however, the qualitative behavior.

The same issue was recently addressed by Liebsch and Wu [85] and also by Hassan and Senechal [86]. There, it is argued that one bath site per correlated cluster site is not sufficient; at least two bath sites per cluster site should be taken into account [86]. Liebsch and Wu disagreed and attributed the opening of the single-particle gap in case of the ringshaped six-site cluster only to the geometry of the cluster and the breaking of translational symmetry in methods such as VCA [85]. We confirm in our analysis that the breaking of translational symmetry is problematic, if not detrimental, for a semimetal state; we explain below, however, that the breaking of translational symmetry affects other clusters as well which do not possess the six-fold rotational symmetry of the six-site cluster. In any case, both Ref. [86] and Ref. [85] agree that the opening of the single-particle gap for infinitesimal $U$, as seen for the six-site cluster, is a numerical artefact of the approach and not physically relevant. Inspired by Ref. [85], we plot the single-particle gap as a function of $U(\lambda=0)$ for various different clusters (Fig. 12). As the main result we observe that the semimetallic phase is never stable with respect to $U$ for the six-site cluster. 
(a)

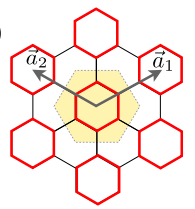

(d)

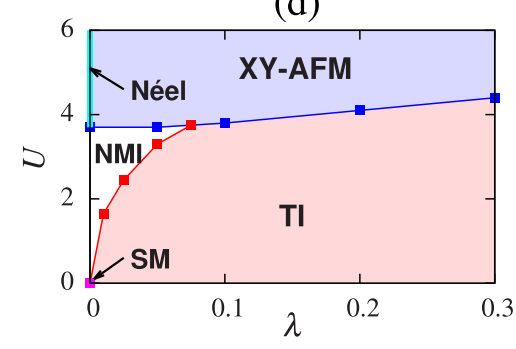

(b)

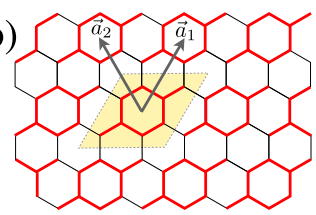

(e)

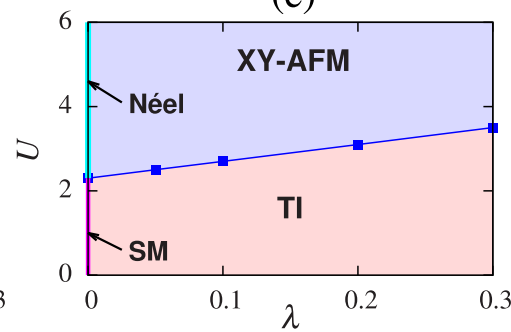

(c)

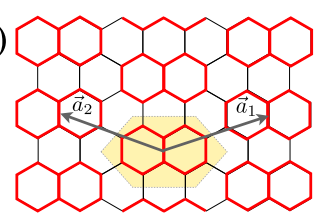

(f)

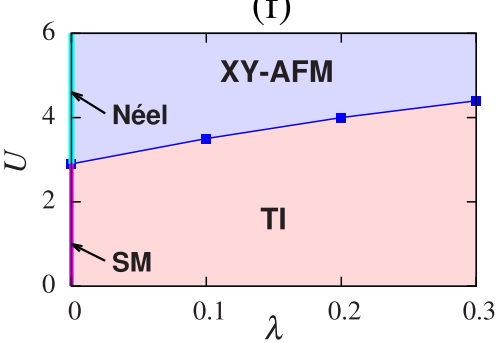

FIG. 11. (Color online) (a)-(c) Coupled-cluster tight-binding scenarios. Red thick links are associated with $\tilde{t}$ and black thin lines with $t$. The second-neighbor spin-orbit links are treated analogously but are omitted for clarity of this figure. (a) Six-site plaquette anisotropic honeycomb lattice. (b) Eight-site lattice and (c) ten-site lattice. (d)-(f) Phase diagram of the Kane-Mele-Hubbard model for different cluster sizes. Note that, in the limit $\lambda=0$, the system displays a magnetic Néel phase and a semimetal phase for all cluster sizes. (d) Six-site cluster. We find a nonmagnetic insulator (NMI), easy-plane antiferromagnetic insulator ( $X Y$-AFM), and topological insulator (TI). The semimetal $(\mathrm{SM})$ only exists for $U=0$. The cyan line indicates the onset of magnetic order $\left(U_{c}=3.8\right.$ for $\left.\lambda=0\right)$. (e) Eight-site cluster. We find SM, TI, and $X Y$-AFM phases. The SM is realized up to $U_{c}=2.4$ where we observe the onset of magnetization. (f) Ten-site cluster. We find SM, TI, and $X Y$-AFM phases. The SM is realized up to $U_{c}=2.9$.

In contrast, the eight- and ten-site clusters seem to provide a stable semimetallic phase up to finite $U_{c}$, which we now study in more detail. None of these clusters exhibit the rotational symmetry of the honeycomb lattice. The eight- and the tensite clusters consists of a single hexagon with two additional "legs" on opposite sites and two hexagons located next to each other, respectively [Figs. 3(b) and 3(c)]. We calculated the band structure with an increased unit cell corresponding to the eight-site cluster. This allows us to take into account the anisotropy. We find that the semimetallic phase present in the isotropic case persists for weak anisotropies. To be more specific, it turns out that the gap does not open; the position of the Dirac cones moves, however, away from the $\boldsymbol{K}$ and $\boldsymbol{K}^{\prime}$ points. (This is understandable, because the three-fold discrete rotation symmetry protects the position of the Dirac cones in

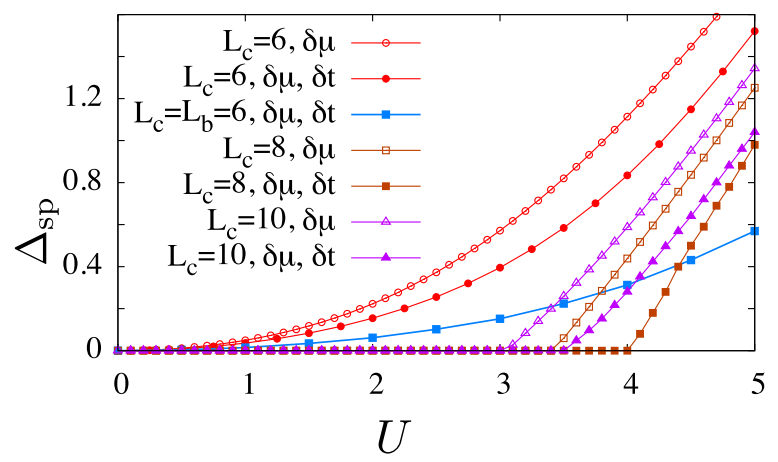

FIG. 12. (Color online) Single-particle gap $\Delta_{\text {sp }}$ as a function of $U(\lambda=0)$ for six-, eight-, and ten-site clusters $\left(L_{c}=6,8,10\right)$ with variation of (i) $\delta \mu$ and (ii) $\delta \mu, \delta t$. In addition, we show $\Delta_{\text {sp }}$ vs $U$ for the six-site cluster with additional bath sites $L_{b}$ (blue curve). Only the paramagnetic solutions, i.e., in the absence of Weiss fields, are displayed. momentum space.) A rather large anisotropy is required to merge the Dirac cones and gap them out. The situation here is reminiscent of the $t_{1}-t_{2}$ model on the honeycomb lattice where a similar behavior is known [87]. By performing a VCA analysis for the eight-site cluster, we find that the semimetallic phase of graphene persists up to $U=2.4$. We also observe within VCA that the position of the Dirac cones is not at $\boldsymbol{K}$ or $\boldsymbol{K}^{\prime}$ anymore, in agreement with the anisotropic band-structure calculation discussed previously $\left(\boldsymbol{K}^{\left({ }^{\prime}\right)}\right.$ refers to the positions of the Dirac cones at $U=0$ ). The phase diagram with additional SO coupling is presented in Fig. 11(e). A similar analysis for the ten-site cluster leads to the same conclusions as for the eight-site cluster [Fig. 11(c)]. Quantitatively, we find a slightly larger $U_{c}=2.9$ where the semimetal-to-Néel-AFM transition occurs [Fig. 11(f)].

\section{Magnetic transition}

Our findings indicate that the symmetric six-site cluster has the smallest tendency towards the formation of magnetic order. The less-symmetric eight-site cluster, in contrast, is significantly more sensitive towards formation of magnetic order and thus underestimates $U_{c}$. This is intuitively clear since the eight-site cluster exhibits two "open legs," i.e., links which have an end site. These end sites are particularly sensitive towards the formation of magnetic order. Ring-shaped clusters such as six- or ten-site clusters, i.e., clusters without end sites, require stronger interactions to acquire magnetic order.

Interestingly, we find that the six-site cluster, while inappropriate for the study of the semimetal phase, is a good choice in order to study magnetism. For the eight-site cluster we can draw the opposite conclusion. The ten-site cluster might be an acceptable compromise; it turns out, however, that for the study with Rashba SO coupling also the ten-site cluster 


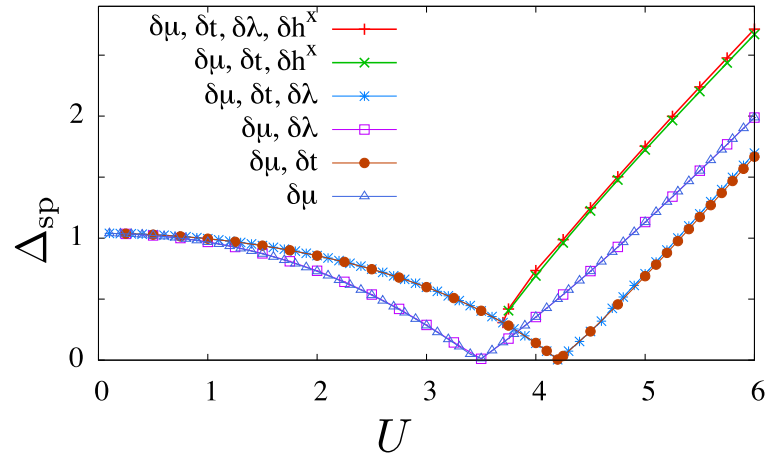

FIG. 13. (Color online) Single-particle gap $\Delta_{\text {sp }}$ as a function of $U$ at $\lambda=0.1$ for the six-site cluster. Different combinations of singleparticle parameters $\left(\delta \lambda, \delta \mu, \delta t\right.$, and Weiss fields $\left.\delta h^{x}\right)$ are varied to yield a saddle-point solution of the grand potential $\Omega$. Varying $\delta h^{x}$, one can see that the single-particle gap does not close at the phase transition between the TI and the $X Y$-AFM phase (red and green curves).

is problematic regarding the investigation of magnetism (see Appendix B for details).

\section{Variation of single-particle parameters}

We briefly discuss the influence of the variation of different single-particle parameters within the VCA. In principle, any single-particle parameter (i.e., $\delta \mu, \delta t, \delta \lambda, \delta \lambda_{R}$ ) can, and should, be varied. Note that the actual value of a single-particle parameter is, e.g., $\mu+\delta \mu$, where $\mu$ is the chosen parameter and $\delta \mu$ comes from the variational scheme. For practical purposes, however, the variation is often restricted to the variation of $\delta \mu$ only. It is then argued that the additional variation of other single-particle parameters does not affect the results anymore. For the six-site cluster, we have already shown for $\lambda=0$ in Fig. 12 that the additional variation of $\delta t$ quantitatively changes the $\Delta_{\mathrm{sp}}$ curve. We also studied this influence for the TI phase at $\lambda=0.1$ for six- and eight-site clusters. In Fig. 13 the single-particle gap $\Delta_{\text {sp }}$ of the six-site cluster is shown for the case where (i) $\delta \mu$ only is varied (dark-blue curve), (ii) $\delta \mu$ and $\delta t$ are varied (dark-red curve), (iii) $\delta \mu$ and $\delta \lambda$ are varied (pink), (iv) $\delta \mu, \delta t$, and $\delta \lambda$ are varied (light blue). Additional variation of the Weiss field $\delta h^{x}$ is also considered for cases (ii) and (iv) (green and red), which reveals that the single-particle gap is not closing at the transition between the TI and the $X Y$-AFM phase [38,71], in agreement with QMC results [36].

Essentially, we find that the additional variation of $\delta t$ is important and has significant effects, which also applies to parameter regimes at finite $\lambda$. It should, hence, be generally taken into account in the variational scheme. The additional variation of $\delta \lambda$, however, might lead to new stationary points but can be neglected because it has only negligible effects (Fig. 14). The same conclusion can be drawn for $\delta \lambda_{R}$. Since the effect of additional variation of $t$ affects all the phases and all the cluster shapes, we find that at least on the honeycomb lattice, one should always vary $\delta \mu$ and $\delta t$ to obtain reliable VCA results.

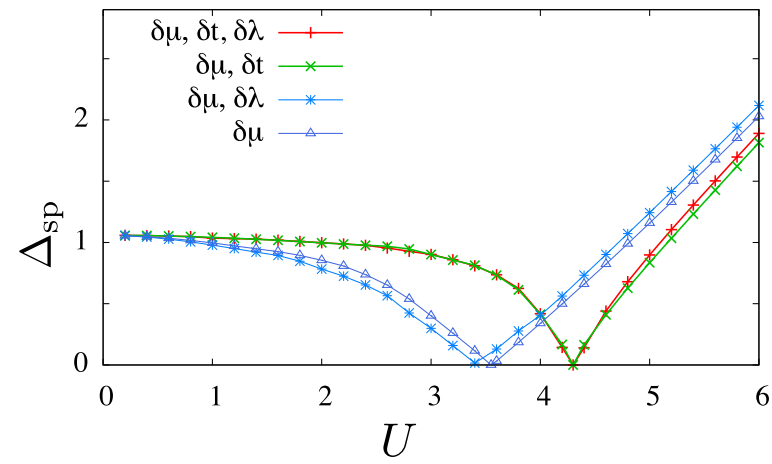

FIG. 14. (Color online) Single-particle gap $\Delta_{\text {sp }}$ as a function of $U$ at $\lambda=0.1$ for the eight-site cluster analogous to Fig. 13 .

\section{APPENDIX B: CLUSTER ANALYSIS OF KMH MODEL

$$
\left(\lambda_{R}>0\right)
$$

\section{Cluster dependence of phase diagram}

In Fig. 15 we show the phase diagram for the eight-site cluster at $\lambda=0.1$. For this parameter, the TS phase is extremely small and very difficult to detect. Therefore, we consider larger intrinsic SO coupling. Figure 16 displays the phase diagrams for the six-, eight-, and ten-site clusters at $\lambda=0.2$. Only for the eight-site cluster (middle panel) we computed edge states which allows us to determine the phase boundary between the TS phase and the metal (red squares). Note that we could likewise perform the analogous computation for armchair edges in the case of six- and ten-site clusters. We do not expect, however, further insights from such an additional computation.

For the eight- and ten-site clusters, calculating the magnetic domain for strong interactions is different from the six-site cluster. The Rashba term acts differently on different links since it depends on $\boldsymbol{\sigma} \times \boldsymbol{d}$. Consequently, the results also depend on the orientation of the cluster. The three different nearest-neighbor links of the honeycomb lattice $\boldsymbol{\delta}_{1}, \boldsymbol{\delta}_{2}$, and $\boldsymbol{\delta}_{3}$ are shown in Fig. 2. It is obvious that a cluster (e.g., the eight-site cluster) which consists of different numbers of $\boldsymbol{\delta}_{1}, \boldsymbol{\delta}_{2}$, and $\boldsymbol{\delta}_{3}$ links, induces a certain anisotropy. Only

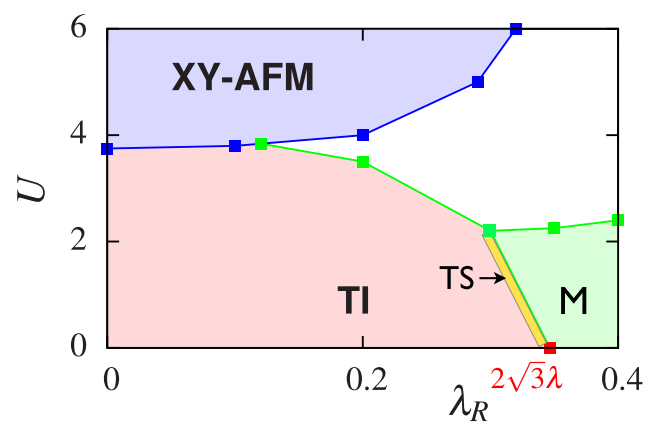

FIG. 15. (Color online) $U-\lambda_{R} \mathrm{KMH}$ phase diagram for $\lambda=0.1$ obtained for an eight-site cluster. In the weak- $\lambda_{R}$ region, only TI and $X Y$-AFM phases exist. The topological-semiconductor (TS) phase is very small for $\lambda=0.1$, but increases with $\lambda$. At larger $\lambda_{R}$ the system is in a metallic phase. In the regime of large $U$ and large $\lambda_{R}$ no magnetic solution commensurate with the eight-site cluster is found. 

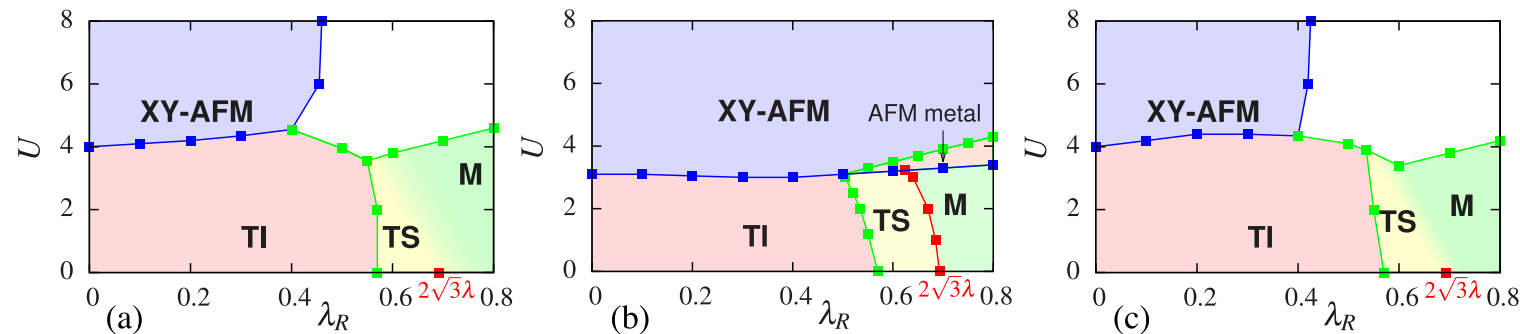

FIG. 16. (Color online) $U-\lambda_{R} \mathrm{KMH}$ phase diagram for $\lambda=0.2$ using the (a) six-site cluster, (b) eight-site cluster, and (c) ten-site cluster. Besides the TI and $X Y$-AFM phase, we find a metal (M) phase (green) and a topological-semiconductor (TS) phase (yellow) which is characterized by the joint occurrence of helical edge states and zero indirect bulk gap. The topological-to-metal phase transition for $U=0$ takes place at $\lambda_{R}=2 \sqrt{3} \lambda$ (yellow-to-green phase). The green boundary is obtained by checking whether (i) the bulk gap is closed via a finite local density of states and whether (ii) edge states are present. At the red boundary, the edge states eventually vanish and one enters a conventional metallic state. For the six- and ten-site clusters we do not find magnetic solutions for $\lambda_{R}>0.4$. For the eight-site cluster, we still find Néel order and an antiferromagnetic metal state characterized by magnetic order and a zero indirect bulk gap (see also Fig. 17).

the ring-shaped six-site cluster exhibits equal numbers of all $\boldsymbol{\delta}_{i}$ links. Therefore, we should consider the results obtained using the six-site cluster as the most reliable reference. Note, however, that we also incorporated the results for eight- and ten-site clusters and eventually argue that the semiquantitative phase diagram should look like Fig. 1.

\section{AFM metal phase and magnetism}

For the eight-site cluster, another interesting situation arises. Even for strong $\lambda_{R}$ and $U$, we find $X Y$-AFM order (for $\lambda=0.1$ and 0.2 ). For $\lambda_{R}>0.5$ and $\lambda=0.2$, however, there is a narrow intermediate- $U$ phase which is an antiferromagnetic metal. Similar to the topological-semiconductor (TS) phase,
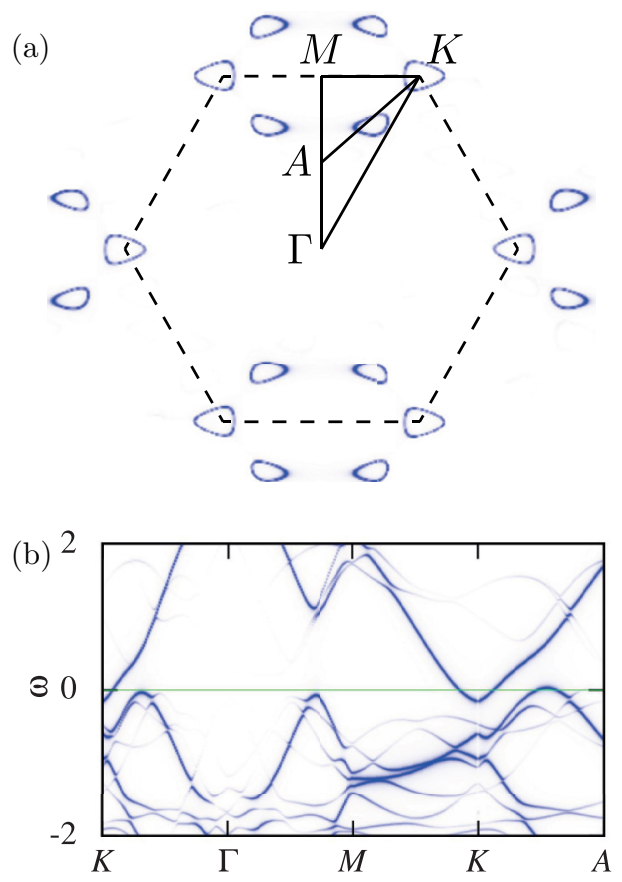

FIG. 17. (Color online) (a) Fermi surface in the AFM metal phase ( $\lambda=0.2, \lambda_{R}=0.6$, and $U=3.3$ ). (b) Single-particle spectral function $A(\boldsymbol{k}, \omega)$ in the AFM metal phase for periodic boundary conditions, plotted along the trajectory shown in panel (a). the strong Rashba coupling bends the bands and gives rise to a metallic density of states. Locally (in momentum space) there is always a direct gap for each wave vector $\boldsymbol{k}$. In contrast to the TS phase, there are no edge states but instead a finite magnetization; thus we call the phase an antiferromagnetic metal. To provide a better understanding of this phase, we show in Fig. 17 the bulk spectral function $A(\boldsymbol{k}, \omega)$ along the path $K \rightarrow \Gamma \rightarrow M \rightarrow K \rightarrow A$. In this plot, one can easily observe that the system is globally gapless, but locally in momentum space there is always a direct gap for each wave vector $\boldsymbol{k}$. We stress that the eight-site cluster exhibits some bias to support such a phase since the onset of magnetization appears for weaker $U$ as compared to other clusters (Fig. 16).

We further find that the antiferromagnetic order loses its $\mathrm{U}(1)$ rotation symmetry in the $x y$ plane. We attribute this effect to the different numbers of $\boldsymbol{\delta}_{1}, \boldsymbol{\delta}_{2}$, and $\boldsymbol{\delta}_{3}$ bonds in the eight-site cluster, which induces anisotropies when Rashba coupling is present. In Fig. 18 we show the grand potential $\Omega$ as a function of $h^{x}$ and $h^{y}$, indicating an antiferromagnetic state pointing in the $y$ direction. We emphasize, however, that changing the orientation of the eight-site cluster also rotates the direction

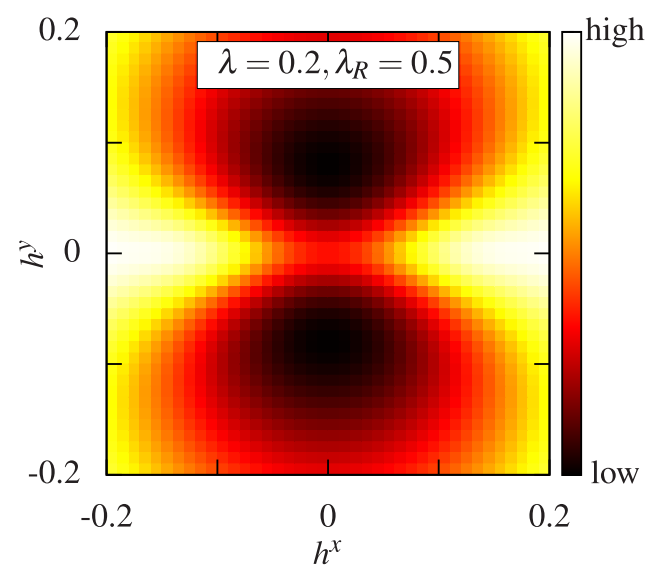

FIG. 18. (Color online) Grand potential heat map as a function of antiferromagnetic Weiss fields, $\Omega\left(h^{x}, h^{y}\right)$ for $\lambda=0.2, \lambda_{R}=0.5$, and $U=4$ on the eight-site cluster. Due to cluster anisotropy, the magnetization points in the $y$ direction. 
of the antiferromagnetic order. This shows that anisotropies in the $x y$ plane are cluster artifacts. We hence conclude that the actual magnetic order is of $X Y$-AFM type. For larger Rashba coupling, we still find magnetic solutions using the eight-site cluster (e.g., the $X Y$-AFM persists up to $\lambda_{R} \sim 1.36$ at $U=8$ ).

The ten-site cluster likewise contains different numbers of $\boldsymbol{\delta}_{i}$ links, leading to similar anisotropies as for the eight-site cluster. Around $\lambda_{R} \sim 0.4$ we observe a breakdown of the magnetic phase (compatible with the results for the six-site cluster). Therefore, we conclude that the resulting VCA phase diagram does not exhibit a magnetically ordered phase for large $\lambda_{R}$ and large $U$ which would be consistent with a magnetic unit cell provided by the small cluster. The aforementioned AFM metal phase, not present for the ten-site cluster, is most likely an artifact of the eight-site cluster and is hence omitted from the final phase diagram in Fig. 1.
[1] C. L. Kane and E. J. Mele, Phys. Rev. Lett. 95, 146802 (2005).

[2] C. L. Kane and E. J. Mele, Phys. Rev. Lett. 95, 226801 (2005).

[3] B. A. Bernevig and S.-C. Zhang, Phys. Rev. Lett. 96, 106802 (2006).

[4] B. A. Bernevig, T. L. Hughes, and S.-C. Zhang, Science 314, 1757 (2006).

[5] M. König, S. Wiedmann, C. Brüne, A. Roth, H. Buhmann, L. W. Molenkamp, X.-L. Qi, and S.-C. Zhan, Science 318, 766 (2007).

[6] M. Z. Hasan and C. L. Kane, Rev. Mod. Phys. 82, 3045 (2010).

[7] X.-L. Qi and S.-C. Zhang, Rev. Mod. Phys. 83, 1057 (2011).

[8] B. A. Bernevig, Topological Insulators and Topological Superconductors (Princeton University Press, Princeton and Oxford, 2013).

[9] H. Min, J. E. Hill, N. A. Sinitsyn, B. R. Sahu, L. Kleinman, and A. H. MacDonald, Phys. Rev. B 74, 165310 (2006).

[10] Y. Yao, F. Ye, X.-L. Qi, S.-C. Zhang, and Z. Fang, Phys. Rev. B 75, 041401 (2007).

[11] C. Weeks, J. Hu, J. Alicea, M. Franz, and R. Wu, Phys. Rev. X 1, 021001 (2011).

[12] C.-C. Liu, W. Feng, and Y. Yao, Phys. Rev. Lett. 107, 076802 (2011).

[13] M. Ezawa, Phys. Rev. Lett. 109, 055502 (2012).

[14] P. Ghaemi, S. Gopalakrishnan, and T. L. Hughes, Phys. Rev. B 86, 201406 (2012).

[15] Y. Xu, B. Yan, H.-J. Zhang, J. Wang, G. Xu, P. Tang, W. Duan, and S.-C. Zhang, Phys. Rev. Lett. 111, 136804 (2013).

[16] A. Shitade, H. Katsura, J. Kuneš, X.-L. Qi, S.-C. Zhang, and N. Nagaosa, Phys. Rev. Lett. 102, 256403 (2009).

[17] M. Jenderka, J. Barzola-Quiquia, Z. Zhang, H. Frenzel, M. Grundmann, and M. Lorenz, Phys. Rev. B 88, 045111 (2013).

[18] D. Xiao, W. Zhu, Y. Ran, N. Nagaosa, and S. Okamoto, Nat. Commun. 2, 596 (2011).

[19] I. Bloch, J. Dalibard, and W. Zwerger, Rev. Mod. Phys. 80, 885 (2008).

[20] P. Soltan-Panahi, J. Struck, P. Hauke, A. Bick, W. Plenkers, G. Meineke, C. Becker, P. Windpassinger, M. Lewenstein, and K. Sengstock, Nat. Phys. 7, 434 (2011).

[21] Y.-J. Lin, R. L. Compton, K. Jimenez-Garcia, J. V. Porto, and I. B. Spielman, Nature (London) 462, 628 (2009).

[22] N. Goldman, I. Satija, P. Nikolic, A. Bermudez, M. A. MartinDelgado, M. Lewenstein, and I. B. Spielman, Phys. Rev. Lett. 105, 255302 (2010).

[23] J. Dalibard, F. Gerbier, G. Juzeliūnas, and P. Öhberg, Rev. Mod. Phys. 83, 1523 (2011).

[24] Y.-J. Lin, K. Jimenez-Garcia, and I. B. Spielman, Nature (London) 471, 83 (2011)

[25] S. Raghu, X.-L. Qi, C. Honerkamp, and S.-C. Zhang, Phys. Rev. Lett. 100, 156401 (2008).
[26] L. Wang, H. Jiang, X. Dai, and X. C. Xie, Phys. Rev. B 85, 235135 (2012).

[27] L. Wang, X. Dai, and X. C. Xie, Europhys. Lett. 98, 57001 (2012).

[28] J. C. Budich, R. Thomale, G. Li, M. Laubach, and S.-C. Zhang, Phys. Rev. B 86, 201407 (2012).

[29] N. A. Garcia-Martinez, A. G. Grushin, T. Neupert, B Valenzuela, and E. V. Castro, Phys. Rev. B 88, 245123 (2013).

[30] M. Daghofer and M. Hohenadler, Phys. Rev. B 89, 035103 (2014).

[31] B. Roy and I. F. Herbut, Phys. Rev. B 88, 045425 (2013).

[32] M. A. N. Araújo, E. V. Castro, and P. D. Sacramento, Phys. Rev. B 87, 085109 (2013).

[33] D. N. Sheng, Z. Y. Weng, L. Sheng, and F. D. M. Haldane, Phys. Rev. Lett. 97, 036808 (2006).

[34] G. E. Volovik, The Universe in a Helium Droplet (Oxford University Press, Oxford, 2003).

[35] S. Rachel and K. Le Hur, Phys. Rev. B 82, 075106 (2010).

[36] M. Hohenadler, T. C. Lang, and F. F. Assaad, Phys. Rev. Lett. 106, 100403 (2011).

[37] D. Soriano and J. Fernández-Rossier, Phys. Rev. B 82, 161302 (2010).

[38] W. Wu, S. Rachel, W.-M. Liu, and K. Le Hur, Phys. Rev. B 85, 205102 (2012).

[39] D. Zheng, G.-M. Zhang, and C. Wu, Phys. Rev. B 84, 205121 (2011).

[40] Y. Yamaji and M. Imada, Phys. Rev. B 83, 205122 (2011).

[41] S.-L. Yu, X. C. Xie, and J.-X. Li, Phys. Rev. Lett. 107, 010401 (2011).

[42] D.-H. Lee, Phys. Rev. Lett. 107, 166806 (2011).

[43] J. Wen, M. Kargarian, A. Vaezi, and G. A. Fiete, Phys. Rev. B 84, 235149 (2011).

[44] M. Mardani, M.-S. Vaezi, and A. Vaezi, arXiv:1111.5980.

[45] M. Hohenadler, Z. Y. Meng, T. C. Lang, S. Wessel, A. Muramatsu, and F. F. Assaad, Phys. Rev. B 85, 115132 (2012).

[46] C. Griset and C. Xu, Phys. Rev. B 85, 045123 (2012).

[47] A. Vaezi, M. Mashkoori, and M. Hosseini, Phys. Rev. B 85, 195126 (2012).

[48] F. F. Assaad, M. Bercx, and M. Hohenadler, Phys. Rev. X 3, 011015 (2013).

[49] Y. Araki and T. Kimura, Phys. Rev. B 87, 205440 (2013).

[50] H.-H. Hung, L. Wang, Z.-C. Gu, and G. A. Fiete, Phys. Rev. B 87, 121113 (2013)

[51] S. Ueda, N. Kawakami, and M. Sigrist, Phys. Rev. B 87, 161108 (2013).

[52] M. H. Zare, F. Fazileh, and F. Shahbazi, Phys. Rev. B 87, 224416 (2013). 
[53] Y. Araki, T. Kimura, A. Sekine, K. Nomura, and T. Z. Nakano, arXiv:1311.3973.

[54] Z. Y. Meng, H.-H. Hung, and T. C. Lang, Mod. Phys. Lett B, 28, 143001 (2014).

[55] H.-H. Hung, V. Chua, L. Wang, and G. A. Fiete, Phys. Rev. B 89, 235104 (2014).

[56] F. F. Assaad and I. F. Herbut, Phys. Rev. X 3, 031010 (2013).

[57] T. Yoshida, S. Fujimoto, and N. Kawakami, Phys. Rev. B 85, 125113 (2012).

[58] T. Yoshida, R. Peters, S. Fujimoto, and N. Kawakami, Phys. Rev. B 87, 085134 (2013).

[59] M. Hohenadler and F. F. Assaad, J. Phys.: Condens. Matter 25, 143201 (2013).

[60] C. Wu, B. A. Bernevig, and S.-C. Zhang, Phys. Rev. Lett. 96, 106401 (2006).

[61] A. Ström, H. Johannesson, and G. I. Japaridze, Phys. Rev. Lett. 104, 256804 (2010).

[62] J. C. Budich, F. Dolcini, P. Recher, and B. Trauzettel, Phys. Rev. Lett. 108, 086602 (2012).

[63] T. Schmidt, S. Rachel, F. von Oppen, and L. I. Glazman, Phys. Rev. Lett. 108, 156402 (2012).

[64] M. Potthoff, M. Aichhorn, and C. Dahnken, Phys. Rev. Lett. 91, 206402 (2003).

[65] M. Potthoff, Eur. Phys. J. B 32, 429 (2003).

[66] J. Reuther, R. Thomale, and S. Rachel, Phys. Rev. B 86, 155127 (2012).

[67] J. Reuther, R. Thomale, and S. Rachel, Phys. Rev. B 90, 100405(R) (2014).

[68] F. D. M. Haldane, Phys. Rev. Lett. 61, 2015 (1988).
[69] D. Cocks, P. P. Orth, S. Rachel, M. Buchhold, K. Le Hur, and W. Hofstetter, Phys. Rev. Lett. 109, 205303 (2012).

[70] P. P. Orth, D. Cocks, S. Rachel, M. Buchhold, K. L. Hur, and W. Hofstetter, J. Phys. B: At., Mol. Opt. Phys. 46, 134004 (2013).

[71] S. Rachel, arXiv:1310.3159.

[72] M. Potthoff, Eur. Phys. J. B 36, 335 (2003).

[73] M. Potthoff, Adv. Solid State Phys. 45, 135 (2005).

[74] D. Sénéchal, P.-L. Lavertu, M.-A. Marois, and A.-M. S. Tremblay, Phys. Rev. Lett. 94, 156404 (2005).

[75] M. Balzer, W. Hanke, and M. Potthoff, Phys. Rev. B 81, 144516 (2010).

[76] V. Gurarie, Phys. Rev. B 83, 085426 (2011).

[77] Z. Wang, X.-L. Qi, and S.-C. Zhang, Phys. Rev. Lett. 105, 256803 (2010).

[78] Z. Wang, X.-L. Qi, and S.-C. Zhang, Phys. Rev. B 85, 165126 (2012).

[79] X.-L. Qi, T. L. Hughes, and S.-C. Zhang, Phys. Rev. B 78, 195424 (2008).

[80] S. Sorella, Y. Otsuka, and S. Yunoki, Sci. Rep. 2, 992 (2012).

[81] M. Kargarian, A. Langari, and G. A. Fiete, Phys. Rev. B 86, 205124 (2012).

[82] T. Liu, B. Douçot, and K. L. Hur, Phys. Rev. B 88, 245119 (2013).

[83] A. Rüegg and G. A. Fiete, Phys. Rev. Lett. 108, 046401 (2012).

[84] K. Seki and Y. Ohta, arXiv:1209.2101.

[85] A. Liebsch and W. Wu, Phys. Rev. B 87, 205127 (2013).

[86] S. R. Hassan and D. Sénéchal, Phys. Rev. Lett. 110, 096402 (2013).

[87] Y. Hasegawa, R. Konno, H. Nakano, and M. Kohmoto, Phys. Rev. B 74, 033413 (2006). 